\title{
MOST FREQUENT GAIT PATTERNS IN DIPLEGIC SPASTIC CEREBRAL PALSY
}

\author{
Mauro César de Morals Filho ${ }^{1-3}$, Cátia Miruki Kawamura ${ }^{1}$, José Augusto Fernandes Lopes ${ }^{1,3}$, Daniella Lins Neves ${ }^{1,3}$, \\ Michelle de Oliveira Cardoso ${ }^{1}$, Jordana Brandão Calafa ${ }^{1}$
}

\begin{abstract}
Objective: To identify gait patterns in a large group of children with diplegic cerebral palsy and to characterize each group according to age, Gross Motor Function Classification System (GMFCS) level, Gait Deviation Index (GDI) and previous surgical procedures. Methods: One thousand eight hundred and five patients were divided in seven groups regarding observed gait patterns: jump knee, crouch knee, recurvatum knee, stiff knee, asymmetric, mixed and non-classified. Results: The asymmetric group was the most prevalent (48.8\%). The jump knee (9.6 years old) and recurvatum (9.4 years old) groups had mean age lower than the other groups. The lowest GDI (43.58) was found in the crouch group. There were more children classified
\end{abstract}

within GMFCS level III in the crouch and mixed groups. Previous surgical procedures on the triceps surae were more frequent in stiff knee and mixed groups. The jump knee group received less and the stiff-knee group more surgical procedures at hamstrings than others. Conclusions: The asymmetrical cases were the most frequent within a group of diplegic patients. Individuals with crouch gait pattern were characterized by the lowest GDI and the highest prevalence of GMFCS III, while patients with stiff knee exhibited a higher percentage of previous hamstring lengthening in comparison to the other groups. Level of Evidence III, Retrospective Comparative Study.

Keywords: Cerebral palsy. Gait. Diplegic spastic.

Citation: Morais Filho MC, Kawamura CM, Lopes JAF, Neves DL, Cardoso MO, Caiafa JB. Most frequent gait patterns in diplegic spastic cerebral palsy. Acta Ortop Bras. [online] 2014;22(4):197-201. Available from URL: http://www.scielo.br/aob.

\section{INTRODUCTION}

The term cerebral palsy $(\mathrm{CP})$ is frequently used to designate a large number of clinical conditions which have in common a specific and non-progressive lesion in the immature brain. ${ }^{1}$ It is related, among other conditions, to movement disorders, such as lack of selective muscle control and muscle imbalance. It is traditionally classified by the clinical type (motor dysfunction) and topography (anatomical region of the lesion). There are some types of motor dysfunction, such as spastic, the most common type, ataxic, hypotonic, dyskinetic (choreo-athetotic, or dystonic) and mixed. The anatomical distribution patterns are generally classified as hemiplegic, diplegic and quadriplegic. Although classifications by motor dysfunction and topography have been widely used, there is no mention about function in it. The Gross Motor Function Classification System (GMFCS) ${ }^{2}$ is a simple, reliable, easily reproducible, and widely used classification based on gross motor function and consists of five distinct functional levels. The classification into levels is based on the patient's usual motor performance, the quality of movement at home and in community settings and the need for assistive technologies. Motor level I includes patients with the least severe motor impairments and motor level $V$ includes patients with the most severe functional limitations. The use of GMFCS has added important information about function in children with $\mathrm{CP}$, but a diversity of gait patterns can be present even in the same functional level. Because of it, many efforts have been made to develop classification or quantification systems for it to assist in clinical diagnosis, decision making and communication. ${ }^{3}$

One of these systems is the Gait Deviation Index (GDI), ${ }^{4}$ which combines information of kinematic data obtained by the three-dimensional motion assessment in the gait laboratory. It allows the characterization of gait in patients by quantifying the overall quality of gait movements using nine kinematic variables. GDI equal to or greater than 100 indicates absence of gait pathologies. Every 10 points below 100 means one standard deviation away from the normal gait pattern. GDI is quantitative and provide an overview of kinematics, but its use is restricted to gait laboratories.

Sutherland and Davids ${ }^{5}$ described four pathological gait patterns based on knee motion in the sagittal plane: jump, recurvatum,

All the authors declare that there is no potential conflict of interest referring to this article.

\footnotetext{
1. Associação de Assistência à Criança Deficiente (AACD), São Paulo, SP, Brazil

2. Instituto de Ortopedia e Traumatologia do Hospital das Clínicas da Faculdade de Medicina da Universidade de São Paulo (IOT/HC/FMUSP), São Paulo, SP, Brazil

3. Instituto de Medicina de Reabilitação do Hospital das Clínicas da Faculdade de Medicina da Universidade de São Paulo (IMREA/HC/FMUSP), São Paulo, SP, Brazil 
crouch and stiff knee. Rodda et al. ${ }^{6}$ classified the gait patterns in spastic diplegic CP into five groups, based on the kinematic analysis in the sagittal plane of the ankle, knee, hip and pelvis. These two classifications systems have similar characteristics, but the classification of Sutherland and Davids ${ }^{5}$ is simpler and easier to apply in a large group of patients.

The purpose of this study was to test the use of Sutherland and Davids classification ${ }^{5}$ in a large group of children with spastic diplegic $\mathrm{CP}$, identifying the prevalence of the four patterns described (jump, recurvatum, crouch and stiff knee). The secondary objective was to characterize each pattern based on age, GMFCS, GDI and surgical history. According to the authors' knowledge, the present study is the first in achieving prevalence and characterization of gait patterns described by Sutherland and Davids ${ }^{5}$ in a large group of children with cerebral palsy.

\section{PATIENTS AND METHODS}

A retrospective cross-sectional study was conducted using the database of the gait laboratory of a tertiary hospital and rehabilitation center, which was approved by the local ethics committee. A search was done for all patients with spastic diplegic CP who underwent gait analysis between 1996 and 2012. Among the 2,928 exams previously selected, only the first examination of each patient was included in the study, for a total of 1,805 patients.

In order to collect kinematic data, reflective markers were strategically placed on specific anatomical landmarks on the participants, as described by Kadaba et al. ${ }^{7}$ The trajectory of the markers within the lab space was captured through an electronic optical system consisting of infrared cameras. Until August 2008, a 6-camera Vicon 370 system $(60 \mathrm{~Hz})$ was used for data capture, and from this date on, an 8-camera QUALISYS OQUS300 system $(500 \mathrm{~Hz})$ was used.

Patients were instructed to walk barefoot in a self-selected speed in an eight-meter walkway (26 feet). A minimum of 10 gait cycles for each assessed leg was collected for consistency evaluation. The data were processed using the software Vicon Clinical Manager (VCM, Oxford Metrics, Oxford, UK) according to the technique described by Davis et al. ${ }^{8}$ Only consistent cases were considered and the analysis was performed from the average of the 10 collected cycles. For data analysis, patients were divided into groups based on the classification proposed by Sutherland and Davids ${ }^{5}$ (jump, crouch, recurvatum, and stiff knee). Patients allocated to the jump-knee group showed the first peak knee flexion above $30^{\circ}$ followed by minimum flexion in single support from $10^{\circ}$ to $20^{\circ}$ in kinematics. Patients classified as crouch knee gait had minimum knee flexion greater than $30^{\circ}$ in stance phase. Patients with recurvatum knee gait showed knee extension below $0^{\circ}$ in stance phase. Individuals classified into the stiff knee gait group exhibited peak knee flexion in swing phase limited to a maximum of $45^{\circ}$ or late peak knee flexion in more than $30 \%$ of the swing phase. Besides these groups, patients were classified into groups exhibiting asymmetrical gait pattern when the knees showed different patterns between right and left sides. Patients were classified as mixed pattern when more than one pattern described by Sutherland and Davids ${ }^{5}$ was observed in the same knee. Finally, the last group consisted of patients who do not fit into any of the foregoing patterns.
The variables age, GMFCS, GDI and previous surgeries (at gastrocnemius-soleus complex, hamstrings and rectus femoris) were analyzed for each individual. We included children with previous surgeries in the study in order to evaluate the potential relationship between the procedure and specific gait patterns. All data were analyzed and comparisons were performed by ANOVA, using the software SPSS V17, Minitab 16 and Office Excel 2010. For comparisons between groups, Tukey's multiple comparison test was applied as well, and the level of significance for all tests was settled on 0.05 (5\%).

\section{RESULTS}

The analysis of 1,805 patients resulted in the following distribution among groups: crouch knee gait with 395 patients (21.88\%); jump knee gait with 168 patients (9.30\%); stiff knee gait with 32 patients (1.77\%); recurvatum knee gait with 70 patients (3.87\%); asymmetrical pattern with 881 patients (48.80\%); mixed pattern with 31 patients (1.70\%) and non-classified group with 228 patients (12.68\%).

Regarding age, the groups with jump and recurvatum knee gait had the lowest means compared with the other groups, with mean ages of 9.0 and 9.4 years old, respectively. There was no significant difference between these two groups as well as among the other groups. (Table 1)

Patients of the crouch knee group had the lowest GDI values (43.58), while the non-classified group exhibited the highest values (64.12). (Table 2) The patients of jump knee (58.91), asymmetrical pattern (56.65), recurvatum knee (56.86) and stiff knee (56.64) groups were not significantly different. The patients of group with mixed pattern showed mean GDI (50.94) lower than those of non-classified and jump knee groups and higher than the crouch group. There was no significant difference among the asymmetric, recurvatum, stiff and jump knee groups.

There were more children classified within GMFCS level III in the mixed and crouch groups (70\% and $57.8 \%$, respectively), and level II in the remaining groups (Jump 50.3\%, Non-Classified 46.6\%, Asymmetrical $44.7 \%$, Stiff $41.9 \%$ and Recurvatum $40 \%$ ). (Table 3 A, B)

The groups with jump and recurvatum knee patterns underwent significantly less triceps surae lengthening procedures than the stiff, crouch, asymmetrical and mixed groups. (Table 4)

The jump knee group showed the lowest percentage of previous hamstrings surgical lengthening, with significantly lower value than the other groups, except for the recurvatum knee group. On the other hand, the stiff knee group exhibited the

\begin{tabular}{|c|c|c|c|c|c|c|c|c|}
\hline Gait pattern & \begin{tabular}{|l|} 
Mean Age \\
(years old)
\end{tabular} & SD & Crouch & Jump & Mixed & NC & Recurv & Stiff \\
\hline Asymmetrical & 11.8 & 6.0 & 0.139 & $<0.001^{*}$ & 0.878 & 0.998 & $0.039^{*}$ & 0.552 \\
\hline Crouch & 12.8 & 6.5 & & $<0.001^{*}$ & 1.000 & 0.826 & $0.001^{*}$ & 0.971 \\
\hline Jump & 9.0 & 8.8 & & & $0.010^{*}$ & $<0.001^{*}$ & \begin{tabular}{|l|l|}
$*$ & 0.999 \\
\end{tabular} & $0.001^{*}$ \\
\hline Mixed & 13.3 & 7.9 & & & & 0.962 & 0.076 & 1.000 \\
\hline Non-classified & 12.1 & 4.7 & & & & & $0.035^{*}$ & 0.754 \\
\hline Recurvatum & 9.4 & 6.7 & & & & & & $0.018^{*}$ \\
\hline Stiff & 13.9 & 6.2 & & & & & & \\
\hline
\end{tabular}


highest percentage of previous hamstrings surgery compared with the other groups, except for the mixed group. (Table 5) The highest percentage of the rectus femoris transfer was observed in the crouch group, with value significantly higher than those of the asymmetrical, jump and non- classifiable groups. (Table 6)

Table 2. Mean GDI according to gait pattern of the studied groups.

\begin{tabular}{c|c|c|c|c|c|c|c|c|c}
\hline Gait pattern & $\begin{array}{c}\text { Mean } \\
\text { GDI }\end{array}$ & SD & Cl & Crouch & Jump & Mixed & NC & Recurv & Stiff \\
\hline Asymmetrical & 56.65 & 13.12 & 0.87 & $<0.001^{*}$ & 0.325 & 0.157 & $<0.001^{*}$ & 1.000 & 1.000 \\
\hline Crouch & 43.58 & 10.04 & 0.99 & & $<0.001^{*}$ & $0.026^{*}$ & $<0.001^{*}$ & $<0.001^{*}$ & $<0.001^{*}$ \\
\hline Jump & 58.91 & 12.39 & 1.87 & & & $0.019^{*}$ & $0.001^{*}$ & 0.912 & 0.965 \\
\hline Mixed & 50.94 & 8.32 & 2.93 & & & & $<0.001^{*}$ & 0.298 & 0.538 \\
\hline Non-classified & 64.12 & 13.99 & 1.82 & & & & & $<0.001^{*}$ & $0.025^{*}$ \\
\hline Recurvatum & 56.86 & 12.64 & 2.96 & & & & & & 1.000 \\
\hline Stiff & 56.64 & 12.60 & 4.37 & & & & & & \\
\hline
\end{tabular}

GDI:gait deviation index; SD:standard deviation; Cl:confidence interval; NC:non-classified; Recurv: recurvatum; * $\mathrm{p}<0.05$

Table 3A. GMFCS distribution according to gait pattern.

\begin{tabular}{c|c|c|c|c|c|c|c|c}
\hline \multirow{2}{*}{ Gait Pattern } & \multicolumn{2}{|c|}{ I } & \multicolumn{2}{|c|}{ II } & \multicolumn{2}{|c|}{ III } & \multicolumn{2}{|c}{ IV } \\
\cline { 2 - 9 } & No. & $\%$ & No. & $\%$ & No. & $\%$ & No. & $\%$ \\
\hline Asymmetrical & 138 & $15.8 \%$ & 391 & $44.7 \%$ & 314 & $35.9 \%$ & 31 & $3.5 \%$ \\
\hline Crouch & 14 & $3.6 \%$ & 98 & $24.9 \%$ & 227 & $57.8 \%$ & 54 & $13.7 \%$ \\
\hline Jump & 37 & $22.2 \%$ & 84 & $50.3 \%$ & 39 & $23.4 \%$ & 7 & $4.2 \%$ \\
\hline Mixed & 1 & $3.3 \%$ & 7 & $23.3 \%$ & 21 & $70.0 \%$ & 1 & $3.3 \%$ \\
\hline Non-classified & 63 & $28.5 \%$ & 103 & $46.6 \%$ & 51 & $23.1 \%$ & 4 & $1.8 \%$ \\
\hline Recurvatum & 15 & $25.0 \%$ & 24 & $40.0 \%$ & 20 & $33.3 \%$ & 1 & $1.7 \%$ \\
\hline Stiff & 4 & $12.9 \%$ & 13 & $41.9 \%$ & 11 & $35.5 \%$ & 3 & $9.7 \%$ \\
\hline
\end{tabular}

\section{DISCUSSION}

In the present study the asymmetrical group was the most frequent $(48.8 \%)$ and only $36.82 \%$ of patients were classified according to patterns described by Sutherland and Davids. ${ }^{5}$ The groups with jump and recurvatum knee gait had the lowest age compared with the other groups, with means of 9.0 and 9.4 years old, respectively. Patients of the crouch knee group had the lowest GDI values (43.58), while the non-classified group exhibited the highest values (64.12). There were more children classified within GMFCS level III in the mixed and crouch groups (70\% and 57.8\%, respectively). The groups with jump and recurvatum knee patterns underwent significantly less triceps surae lengthening procedures and the highest percentage of the rectus femoris transfer was observed in the crouch group. The jump knee group showed the lowest percentage of previous hamstrings surgical lengthening and the stiff knee group exhibited the highest.

Rodda et al. ${ }^{9}$ also found a substantial number of patients with asymmetrical impairments between sides and designed a classification system according to involved limbs, not by individual patients, in a longitudinal study on the natural progression of the disease. The present study has a cross-sectional design and it did not evaluate the natural history. The main purpose was to identify the prevalence of most frequent patterns in a large group of CP patients and the design applied is appropriate for this study.

The group of patients who did not fit in any of the primary classifications (12.68\%) was apparently characterized by less severe impairments, exhibiting the highest GDI and prevalence of GMFCS II. The GDI combines information of kinematic data obtained by the three-dimensional motion assessment in the gait laboratory. It allows the characterization of gait in patients by quantifying the overall quality of gait movements using nine

Table 3B. Tukey's multiple comparison test.

\begin{tabular}{|c|c|c|c|c|c|c|c|}
\hline & & Asymmetrical & Crouch & Jump & Mixed & Non-classified & Recurvatum \\
\hline \multirow{6}{*}{ I } & Crouch & $<0.001$ & & & & & \\
\hline & Jump & 0.044 & $<0.001$ & & & & \\
\hline & Mixed & 0.063 & 0.948 & 0.016 & & & \\
\hline & Non-classified & $<0.001$ & $<0.001$ & 0.157 & 0.003 & & \\
\hline & Recurvatum & 0.062 & $<0.001$ & 0.653 & 0.011 & 0.591 & \\
\hline & Stiff & 0.664 & 0.013 & 0.243 & 0.173 & 0.066 & 0.178 \\
\hline \multirow{6}{*}{$\|$} & Crouch & $<0.001$ & & & & & \\
\hline & Jump & 0.186 & $<0.001$ & & & & \\
\hline & Mixed & 0.020 & 0.845 & 0.006 & & & \\
\hline & Non-classified & 0.618 & $<0.001$ & 0.471 & 0.016 & & \\
\hline & Recurvatum & 0.475 & 0.014 & 0.171 & 0.117 & 0.362 & \\
\hline & Stiff & 0.758 & 0.038 & 0.392 & 0.122 & 0.625 & 0.859 \\
\hline \multirow{6}{*}{ III } & Crouch & $<0.001$ & & & & & \\
\hline & Jump & 0.002 & $<0.001$ & & & & \\
\hline & Mixed & $<0.001$ & 0.190 & $<0.001$ & & & \\
\hline & Non-classified & $<0.001$ & $<0.001$ & 0.949 & $<0.001$ & & \\
\hline & Recurvatum & 0.685 & $<0.001$ & 0.131 & $<0.001$ & 0.105 & \\
\hline & Stiff & 0.960 & 0.016 & 0.153 & 0.007 & 0.133 & 0.837 \\
\hline \multirow{6}{*}{ IV } & Crouch & $<0.001$ & & & & & \\
\hline & Jump & 0.684 & $<0.001$ & & & & \\
\hline & Mixed & 0.950 & 0.102 & 0.826 & & & \\
\hline & Non-classified & 0.190 & $<0.001$ & 0.162 & 0.575 & & \\
\hline & Recurvatum & 0.439 & 0.008 & 0.363 & 0.613 & 0.941 & \\
\hline & Stiff & 0.078 & 0.523 & 0.200 & 0.317 & 0.013 & 0.077 \\
\hline
\end{tabular}


Table 4. Incidence of previous plantar flexor lengthening according to gait pattern of the studied groups.

\begin{tabular}{|c|c|c|c|c|c|c|c|c|}
\hline \multirow{2}{*}{$\begin{array}{l}\text { Triceps surae } \\
\text { lengthening }\end{array}$} & \multicolumn{2}{|c|}{ Yes } & \multirow{2}{*}{ Crouch } & \multirow{2}{*}{ Jump } & \multirow{2}{*}{ Mixed } & \multirow{2}{*}{ Non- Classified } & \multirow{2}{*}{ Recurvatum } & \multirow{2}{*}{ Stiff } \\
\hline & No. & $\%$ & & & & & & \\
\hline Asymmetrical & 365 & $41.4 \%$ & 0.479 & $<0.001^{*}$ & $0.028^{*}$ & 0.105 & $0.005^{*}$ & 0.188 \\
\hline Crouch & 172 & $43.5 \%$ & & $<0.001^{*}$ & 0.056 & $0.050^{*}$ & $0.002^{*}$ & 0.294 \\
\hline Jump & 41 & $24.4 \%$ & & & $<0.001^{*}$ & $0.018^{*}$ & 0.984 & $0.001^{*}$ \\
\hline Mixed & 19 & $61.3 \%$ & & & & $0.006^{*}$ & $<0.001^{*}$ & 0.513 \\
\hline Non-classified & 81 & $35.5 \%$ & & & & & 0.080 & 0.054 \\
\hline Recurvatum & 17 & $24.3 \%$ & & & & & & $0.004^{*}$ \\
\hline Stiff & 17 & $53.1 \%$ & & & & & & \\
\hline
\end{tabular}

${ }^{\star} p<0.05$

Table 5. Incidence of previous hamstring lengthening according to gait pattern of the studied groups

\begin{tabular}{|c|c|c|c|c|c|c|c|c|}
\hline \multirow{2}{*}{$\begin{array}{c}\text { Hamstring } \\
\text { lengthening }\end{array}$} & \multicolumn{2}{|c|}{ Yes } & \multirow{2}{*}{ Crouch } & \multirow{2}{*}{ Jump } & \multirow{2}{*}{ Mixed } & \multirow{2}{*}{ Non- Classified } & \multirow{2}{*}{ Recurvatum } & \multirow{2}{*}{ Stiff } \\
\hline & No. & $\%$ & & & & & & \\
\hline Asymmetrical & 259 & $29.4 \%$ & $0.020^{\star}$ & $<0.001^{*}$ & 0.060 & $0.048^{*}$ & $0.007^{\star}$ & $<0.001^{*}$ \\
\hline Crouch & 142 & $35.9 \%$ & & $<0.001^{*}$ & 0.305 & $<0.001^{*}$ & $<0.001^{*}$ & $0.003^{*}$ \\
\hline Jump & 17 & $10.1 \%$ & & & $<0.001^{*}$ & $0.001^{*}$ & 0.356 & $<0.001^{*}$ \\
\hline Mixed & 14 & $45.2 \%$ & & & & $0.007^{*}$ & $<0.001^{*}$ & 0.167 \\
\hline Non-classified & 52 & $22.8 \%$ & & & & & 0.124 & $<0.001^{*}$ \\
\hline Recurvatum & 10 & $24.3 \%$ & & & & & & $<0.001 *$ \\
\hline Stiff & 20 & $53.1 \%$ & & & & & & \\
\hline
\end{tabular}

${ }^{*} p<0.05$.

Table 6. Incidence of previous rectus femoris transfer surgery according to gait pattern of the studied groups.

\begin{tabular}{|c|c|c|c|c|c|c|c|c|}
\hline \multirow{2}{*}{$\begin{array}{l}\text { Rectus femoris } \\
\text { transfer }\end{array}$} & \multicolumn{2}{|c|}{ Yes } & \multirow{2}{*}{ Crouch } & \multirow{2}{*}{ Jump } & \multirow{2}{*}{ Mixed } & \multirow{2}{*}{ Non- Classified } & \multirow{2}{*}{ Recurvatum } & \multirow{2}{*}{ Stiff } \\
\hline & No. & $\%$ & & & & & & \\
\hline Asymmetrical & 29 & $3.3 \%$ & $0.007^{\star}$ & 0.298 & 0.984 & 0.391 & 0.657 & 0.297 \\
\hline Crouch & 26 & $6.6 \%$ & & $0.018^{*}$ & 0.460 & $0.015^{*}$ & 0.464 & 0.134 \\
\hline Jump & 3 & $1.8 \%$ & & & 0.600 & 0.776 & 0.262 & 0.446 \\
\hline Mixed & 1 & $3.2 \%$ & & & & 0.720 & 0.801 & 0.306 \\
\hline Non-classified & 5 & $2.2 \%$ & & & & & 0.343 & 0.398 \\
\hline Recurvatum & 3 & $4.3 \%$ & & & & & & 0.235 \\
\hline
\end{tabular}

${ }^{*} p<0.05$.

kinematic variables. GDI equal to or greater than 100 indicates absence of gait pathologies. Every 10 points below 100 means one standard deviation away from the normal gait pattern. ${ }^{4}$ Lin et al..$^{10}$ also found a group of patients showing a better gait pattern with absence of specific kinematics changes of the knee, which highlights the need for more detailed classifications for this group of patients as well.

The group of patients in which more than one pattern, of those described by Sutherland and Davids, ${ }^{5}$ was observed in the same knee is apparently characterized by relatively more severe impairments, with the second lowest GDI and prevalence of patients with GMFCS III. The stiff-knee gait pattern is the only one which refers to changes in the knee motion during the swing phase and may be present as part of another pathological knee pattern during the stance phase, therefore, it should not be regarded as a single and individual pattern. ${ }^{5}$

The second largest group observed in this study is the crouch gait group, which is in agreement with results obtained by Wren et al. ${ }^{11}$ in 2004. In that study, however, the highest prevalence was the stiff-knee gait pattern. It is the group of patients with the most severe impairment, which GDI was the lowest, with prevalence of GMFCS III patients. The highest incidence of previous rectus femoris transfer was observed in this group. Although previous studies have shown that the rectus femoris muscle does not actively participate in the knee extension during the stance phase of the normal gait, ${ }^{12,13}$ other studies demonstrate increased knee flexion after surgery in the long term. ${ }^{14-16}$

The groups jump and recurvatum knee were formed by the youngest patients, which is in accordance to the description given by Sutherland and Davids. ${ }^{5}$ These authors associate both the jump and the recurvatum knee to the triceps surae spasticity or contracture. They are also the patients with the lowest number of previous surgeries, as described by Wren et al., ${ }^{11}$ who reported the effectiveness of surgeries, in general, in reducing the likelihood of having ankle equinus.

The group of patients with exclusively stiff knee pattern during swing phase had the highest incidence of previous surgeries. Patients in the group of stiff gait pattern showed the highest rate of hamstring lengthening; however, none of them underwent rectus femoris transfer. The co-spasticity of the hamstrings and quadriceps in the swing phase is very common ${ }^{15}$ and the masking of the rectus femoris muscle dysfunction by hamstring shortening was also described by Wren et al. ${ }^{11}$ in 2004. The group with stiff knee gait pattern also had one of the highest 
rates of triceps surae lengthening (53.1\%). Although many authors do not agree with the role of plantar flexors in propulsion generation (i.e., forward acceleration of the mass center), ${ }^{17}$ there are new studies describing that elastic energy is stored in the tendon along the plantar flexors when they are stretched to the maximum at the end of the single support. This energy causes a rapid plantar flexion of the ankle in pre-swing, helps pushing the tibia forward and thus contributes to approximately $40^{\circ}$ of knee flexion required at this stage of the cycle. ${ }^{18} \mathrm{~A}$ possible weakness caused by plantar flexor lengthening can then interfere with this phenomenon, currently known as the fourth rocker mechanism, and lead to delay and limitation of peak knee flexion in the swing phase.

There was no relationship between the hyperextension of the knee and prior stretching of the hamstring. Based on this, we believe that the hyperextension of the knee in the studied sample is rather primary than iatrogenic.

The present study has some limitations. As described, it is a cross-sectional study, designed in order to determine the prevalence of most frequent gait patterns in a large group of cerebral palsy patients and characterize them. The study does not provide information about natural history and the inclusion of patients with previous surgeries can be a confounding factor, however the medical history of each patient, including previous surgeries, is an important issue for the characterization of groups. As observed in the present data, specific gait patterns exhibited relation with previous surgery and these findings should be investigated in the future using a different study design. On the other hand, the results have significant clinical implications. The determination of most frequent gait patterns prevalence in a population is important for planning treatment approaches and resources. In addition to this, when gait analysis was applied, a substantial number of asymmetrical patients were identified, even in a group of diplegic spastic CP. This information should be considered during a decision making process in clinical setting, because part of treatment indications can be asymmetrical, even in children with diplegic spastic CP.

\section{CONCLUSION}

In conclusion, only $36.82 \%$ of the studied patients were classified according to the classic patterns. The asymmetrical cases were more frequent within a group of diplegic patients. Individuals with crouch gait pattern were characterized by the lowest GDI, the highest prevalence of GMFCS III and previous rectus femoris transfer, while patients with stiff knee exhibited a higher percentage of previous hamstring lengthening in comparison with other groups.

\section{ACKNOWLEDGEMENTS}

We thank Mr. Jimmy Adans Costa Palandi for statistical analysis and Mr. Ilson Ribeiro Soares for support and kind attention during data collection.

\section{REFERENCES}

1. Rosenbaum P, Paneth N, Leviton A, Goldstein M, Bax M, Damiano D, et al. A report: the definition and classification of cerebral palsy April 2006. Dev Med Child Neurol Suppl. 2007;109:8-14.

2. Palisano RJ, Hanna SE, Rosenbaum PL, Russell DJ, Walter SD, Wood EP, et al. Validation of a model of gross motor function for children with cerebral palsy. Phys Ther. 2000;80(10):974-85

3. Dobson F, Morris ME, Baker R, Graham HK. Gait classification in children with cerebral palsy: a systematic review. Gait Posture. 2007;25(1):140-52.

4. Schwartz MH, Rozumalski A. The Gait Deviation Index: a new comprehensive index of gait pathology. Gait Posture. 2008;28(3):351-7.

5. Sutherland DH, Davids JR. Common gait abnormalities of the knee in cerebral palsy. Clin Orthop Relat Res. 1993;(288):139-47.

6. Rodda JM, Graham HK, Carson L, Galea MP, Wolfe R. Sagittal gait patterns in spastic diplegia. J Bone Joint Surg Br. 2004;86(2):251-8.

7. Kadaba MP, Ramakrishnan HK, Wootten ME. Measurement of lower extremity kinematics during level walking. J Orthop Res. 1990;8(3):383-92.

8. Davis RB, Ôunpuu S, Tyburski DJ, Gage JR. A gait analysis data collection and reduction technique. Hum Mov Sci. 1991;10:575-87.

9. Rodda J, Graham HK. Classification of gait patterns in spastic hemiplegia and spastic diplegia: a basis for a management algorithm. Eur J Neurol. 2001;8(Suppl 5):98-108.

10. Lin CJ, Guo LY, Su FC, Chou YL, Cherng RJ. Common abnormal kinetic patterns of the knee in gait in spastic diplegia of cerebral palsy. Gait Posture. 2000;11(3):224-32
11. Wren TA, Rethlefsen S, Kay RM. Prevalence of specific gait abnormalities in children with cerebral palsy: influence of cerebral palsy subtype, age, and previous surgery. J Pediatr Orthop. 2005;25(1):79-83.

12. Nene A, Byrne $C$, Hermens $H$. Is rectus femoris really a part of quadriceps? Assessment of rectus femoris function during gait in able-bodied adults. Gait Posture. 2004;20(1):1-13.

13. Arnold AS, Anderson FC, Pandy MG, Delp SL. Muscular contributions to hip and knee extension during the single limb stance phase of normal gait: a framework for investigating the causes of crouch gait. J Biomech. 2005;38(11):2181-9

14. Saw A, Smith PA, Sirirungruangsarn Y, Chen S, Hassani S, Harris G, Kuo KN Rectus femoris transfer for children with cerebral palsy: long-term outcome. J Pediatr Orthop. 2003;23(5):672-8.

15. Carney BT, Oeffinger D, Gove NK. Sagittal knee kinematics after rectus femoris transfer without hamstring lengthening. J Pediatr Orthop. 2006;26(2):265-7.

16. Rethlefsen SA, Kam G, Wren TA, Kay RM. Predictors of outcome of distal rectus femoris transfer surgery in ambulatory children with cerebral palsy. $J$ Pediatr Orthop B. 2009;18(2):58-62.

17. Sutherland $\mathrm{DH}$, Cooper L, Daniel D. The role of the ankle plantar flexors in normal walking. J Bone Joint Surg Am. 1980;62(3):354-63.

18. Perry J, Burnfield J. Gait analysis: normal and pathological function. 2nd ed Thorofare: Slack Inc.; 2010. 